\title{
Conocimientos y prácticas frente al Chikungunya Barcelona, Quindío, 2016
}

\author{
Knowledge and practices about Chikungunya in Barcelona, \\ Quindío, Colombia, 2016
}

\author{
Carmen Luisa Betancourt Pulgarín', Víctor Daniel Calvo Betancur², Diana Marcela González ${ }^{3}$, Alejandra Ordóñez Molina ${ }^{4}$, \\ Diana María Romero Flórez ${ }^{5}$
}

\author{
Para citar este artículo: Betancourt CL, Calvo VD, González DM, Ordóñez A, Romero DM. Conocimientos y prácticas frente al chikungunya \\ Barcelona, Quindío, 2016. Ustasalud. 2017;16: 43-51
}

Licencia Creative Commons

\section{(c) (1) () $\Theta$} lo tanto, los lectores pueden acceder libremente a los artículos en su formato .pdf, igualmente podrán descargarlos y difundirlos; sin embargo no podrán modificarlos o alterarlos, adicionalmente se debe reconocer la autoría de las personas que figuran en las publicaciones, pero estas no podrán comercializadas.

\section{RESUMEN}

Objetivo: Determinar los conocimientos y prácticas acerca del chikungunya en el corregimiento de Barcelona, departamento del Quindío en el primer trimestre del 2016.

Metodología: Estudio de tipo descriptivo, transversal. Para la recolección de datos se aplicó una encuesta estructurada, la cual incluye una sección de datos sociodemográficos, una sección de conocimientos y una de prácticas. En total se aplicó la encuesta a 277 individuos que previamente firmaron consentimiento informado.

Resultados: La población de los barrios objeto de estudio reportó un inadecuado conocimiento sobre la enfermedad, además de una deficiente implementación de prácticas, lo que hace que el corregimiento de Barcelona sea un área de importancia para la implementación de programas de salud pública y demás afines.

Conclusiones: Las encuestas tipo CAP permiten evaluar objetivamente las poblaciones, para crear programas y elaborar planes con el fin de mejorar la calidad de vida de los individuos y las poblaciones por intervenir, y aumentar la participación comunitaria.

Palabras clave: Chikungunya, epidemiología, salud pública.

\section{ABSTRACT}

Objective: The aim of this study is determine knowledge and practices about chikungunya in the district of Barcelona Quindío at the first trimester of 2016.

Methodology: A cross-sectional descriptive study. The data was collected using a structured questionnaire, including sociodemographic, knowledge and practical aspects or items. The survey was applied on 277 individuals which previously get the consent with proper signature.

Results: Survey it was found an inadequate knowledge about the disease, besides insufficient implementation of practices which become the Barcelona district as an important area to intervene and develop public health programs.

Conclusions: The knowledge, attitudes and practices questionnaries allow to assess, in populations an objective way of population, leading to develop programs with the aim of intervene into vulnerable population, increase community participation and enhance quality of life of individuals.

Keywords: Chikungunya, epidemiology, public health.
1 Grupo de investigación ZAFETI, Facultad Ciencias de la Salud, Fundación Universitaria del Área Andina, Pereira. Grupo de Investigación Vulnerabilidad y Salud Pública, Facultad de Medicina, Universidad Tecnológica de Pereira, Pereira, Colombia

2 Grupo de investigación Alergología clínica y experimental (GACE), Facultad de Medicina, Universidad de Antioquia, Medellín, Colombia

${ }^{3}$ Secretaría Departamental de Salud Quindío, Armenia, Colombia

${ }^{4}$ Escuela de Odontología, Universidad del Valle, Cali, Colombia

${ }^{5}$ Facultad de Medicina, Universidad de Cartagena, Cartagena, Colombia

Autor de correspondencia: Alejandra Ordoñez Molina Correo electrónico: amom0108@gmail.com

Recibido para publicación:

6 de junio de 2017 Aceptado para publicación: 21 de junio de 2017 


\section{INTRODUCCIÓN}

El chikungunya es una enfermedad viral que se propaga por la picadura de los mosquitos Aedes aegypti o Aedes albopictus, este último, en menor frecuencia ${ }^{1,2}$. Esta enfermedad afecta a los grupos de población de todas las edades y niveles socioeconómicos, haciéndose presente en más de 60 países de África, Asia, Europa y las Américas ${ }^{3}$.

Los organismos que trasportan el virus del chikungunya (CHIKV) emergen debido a cambios ecológicos, climáticos y ambientales que permiten que sean invasores de nuevos nichos como las urbes humanas. Tienden a crecer en ambientes hostiles, pero se benefician de microclimas tropicales con alturas por debajo de los $1800 \mathrm{msnm}$ donde puedan sobrevivir en estado de huevo, larva y estados maduros ${ }^{4-7}$.

Según datos de la Organización Mundial de la Salud para el 2015, la enfermedad se consolidó como endémica en los países del hemisferio occidental, con cifras que alcanzaron los 693.489 casos sospechosos y 37.480 confirmados en todo el continente americano. Colombia ocupó el primer puesto en reportes a la Organización Panamericana de la Salud (OPS) con cifras de 356.079 casos, lo cual generó en el país la necesidad de tener disponibles los recursos económicos girados para la atención de aproximadamente \$ 6.408.723.000 COP según Resolución 3940/2014.

Por parte, del Instituto Nacional de Salud se notificaron hasta la semana 52 del 2015359.728 casos de $(\mathrm{CHIKV})^{8}$. El acumulado de casos notificados al Sistema Nacional de Vigilancia en Salud Publica (SIVIGILA) hasta la semana epidemiológica 15 de 2016 es de 12.598, comparado con la semana epidemiológica 15 de 2015 en la cual se notificaron 11.991 casos del evento. El promedio de casos notificados por semana hasta la semana 15 de 2016 fue de $839^{9}$.

La investigación se realizó en el corregimiento de Barcelona, Quindío, el cual se caracteriza por tener un clima cálido, con pocas lluvias, lo que lo convierte en un lugar propicio para la propagación del vector de la enfermedad. El corregimiento más grande del país a pesar de ello, es dependiente económica y políticamente del municipio de Calarcá, lo que lo hace vulnerable al impacto de las arbovirosis emergentes, ya que sus programas de prevención, control y erradicación de los vectores no se han implementado. Quindío a pesar de ser el departamento más pequeño de Colombia reportó 5908 casos para el 2015, al informe epidemiológico de la semana 15 del 2016 el departamento notificó 328 casos, ubicándose en el puesto 12 a nivel nacional ${ }^{10}$.

Para los proveedores de atención de salud en las Américas, el virus del chikungunya era una enfermedad relacionada con los viajeros, de baja frecuencia, pero, en la actualidad se ha convertido en una enfermedad endémica en el hemisferio occidental con informes de casos autóctonos desde la Florida hasta América del Sur superior ${ }^{11}$.

Dicho escenario obligó a la creación de políticas, planes de gestión y la disponibilidad de los recursos por parte del Gobierno. El Instituto Nacional de Salud (INS) generó protocolos de atención, así como campañas de información por medios de comunicación masiva ${ }^{12}$.

A pesar de los esfuerzos, no se alcanzó el impacto deseado, dado que el contagio continúa y el mosquito se sigue propagando. Lo anterior puede relacionarse con la falta de información a la comunidad, o también, al desinterés o la falta de voluntad de aplicar los conocimientos para disminuir el riesgo de adquirir el chikungunya ${ }^{13}$.

El grado de conocimiento de una comunidad ante un evento y su capacidad para aplicarlo puede medirse mediante una encuesta tipo conocimientos, actitudes y prácticas CAP, además permite evaluar el impacto de una enfermedad en la población ${ }^{14}$.

La situación descrita da al equipo de investigación la necesidad de determinar los conocimientos y prácticas, frente a la infección del virus del chikungunya, de la población del corregimiento de Barcelona, Quindío, primer semestre 2016.

\section{METODOLOGÍA}

Se realizó un estudio de tipo descriptivo de corte transversal, en la población de Barcelona (Calarcá, Quindío). Se escogieron a conveniencia los barrios 
de Congal I, Congal II y Álamos por presentar la mayor incidencia de la enfermedad ${ }^{15}$, se hizo un censo en los barrios seleccionados para establecer de forma exacta el número de casas. El censo mostró 281 casas para los tres barrios, por lo que se decidió tomar el total de la población seleccionada de las cuales no hubo respuesta en 4 casas por lo que no fueron incluidas en el estudio.

Para la recolección de la información se empleó una encuesta estructurada para medir el nivel de conocimiento y su aplicación. Las preguntas se enviaron a validación por parte de expertos, donde se evaluó la pertinencia, el grado de aporte a la investigación, la redacción y estructura. Además, se realizó una prueba piloto en corregimiento en una zona diferente, a esta se le aplicó el instrumento final, pero con condiciones sociodemográficas similares, de la cual se modificó también el instrumento.

Para realizar la encuesta se incluyeron personas con la capacidad física y mental de responderla, debían tener 18 años de edad o más, debían encontrarse en casa al momento de la visita y aceptar responder la encuesta; no se excluyeron individuos.

La encuesta se realizó a una sola persona de la familia. Al momento de finalizar la encuesta se hizo la entrega de un plegable informativo que incluía la descripción de la enfermedad, los síntomas, las medidas preventivas y las acciones para realizar una vez se sospechara la presencia de la enfermedad.

\section{Análisis estadísticos}

Los datos fueron recolectados por un equipo entrenado bajo la supervisión y validación de los investigadores. Se tabuló la información en una base de datos en Microsoft Excel ${ }^{\bullet}$ (Excel 2013; Microsoft Corporation, Redmont: WA, USA) con la validación del software Epi Info $^{\text {tw }} 6.04$ (CDC, Atlanta, GA, USA, 2011). Las preguntas se clasificaron como correctas (0) e incorrectas (1). Las categorías de conocimiento y prácticas se convirtieron en dicotómicas de la siguiente manera; se agruparon las preguntas de cada variable y se denominó tiene conocimiento (0) o buenas prácticas (0) si el individuo respondía correctamente el $80 \%$ de las preguntas. Los análisis se desarrollaron en el paquete estadístico Stata ${ }^{\circledR}$ 13.0. (Stata Corp. 2013 Stata statistical sofware: Release 13. College Stations, TX: Stata Corp LP. Se hicieron análisis descriptivos univariados con medias y desviación estándar, además, un análisis bivariado para relacionar los conocimientos y prácticas con la aplicación de la prueba de $\mathrm{X}^{2}$ y la obtención de OR para determinar qué características pueden actuar como factores protectores o de riesgo.

\section{Aspectos éticos}

La investigación se clasificó como sin riesgo según Resolución 8430/1993. Se aplican los principios de autonomía y beneficencia, reconociendo la declaración de Helsinki en la cual el respeto por el individuo es uno de los principios básicos, mediante la presentación de un consentimiento informado, para el ingreso al estudio y solo se aplicó a mayores de 18 años.

\section{Compromiso medioambiental}

Se evitó emplear medios impresos para el manejo de la información, a excepción de las encuestas, las cuales se imprimieron en papel ecológico de caña de azúcar.

\section{RESULTADOS}

La muestra evaluada fue de 277 individuos de un total de 281 viviendas en los barrios seleccionados, se eliminaron cuatro viviendas por no encontrar respuesta.

Respecto a los factores sociodemográficos se halló que el $99 \%$ de las personas encuestadas se reconocen como mestizas, el 59,6\% se dedicaban a labores del hogar y el $17 \%$ refirieron ser trabajadores independientes. Todas las viviendas tenían servicios de agua, luz, alcantarillado y recolección de basuras y el $97 \%$ de la población contaba con calles pavimentadas (Tabla 1).

El 44\% de la población encuestada refirió haber conocido de casos de chikungunya en su barrio en los últimos seis meses, mientras el $86 \%$ de los encuestados refirió que la trasmisión de la enfermedad se da por la picadura del mosquito y el $63,5 \%$ refirió que el mosquito pica durante todo el día. El 49\% refirió que la larva es un gusano y el $25 \%$ manifestó que es un huevo. Frente a las acciones que conoce la población para prevenir la enfermedad el $17,7 \%$ de la población reconoció la eliminación de aguas estancadas 
como acción de prevención, mientras solo el $0,4 \%$ de la población consideró el uso del toldillo como acción preventiva.

Tabla 1. Características sociodemográficas en el corregimiento de Barcelona, departamento del Quindío, 2016

\begin{tabular}{|c|c|c|}
\hline Característica & Frecuencia & Porcentaje \\
\hline \multicolumn{3}{|l|}{ Cabeza de familia } \\
\hline Sí & 209 & 75,5 \\
\hline No & 68 & 24,6 \\
\hline \multicolumn{3}{|l|}{ Sexo } \\
\hline Femenino & 220 & 80,1 \\
\hline Masculino & 57 & 20,6 \\
\hline Edad (mediana, rango) & 43,2 & $18-89$ \\
\hline \multicolumn{3}{|l|}{ Escolaridad } \\
\hline Primaria incompleta & 65 & 23,5 \\
\hline Primaria completa & 44 & 15,9 \\
\hline Secundaria incompleta & 56 & 20,2 \\
\hline Secundaria completa & 62 & 22,4 \\
\hline Técnico o tecnólogo & 14 & 5,1 \\
\hline Universitario & 8 & 2,9 \\
\hline Posgrado & 1 & 0,4 \\
\hline Ninguno & 27 & 9,8 \\
\hline \multicolumn{3}{|l|}{ Estrato social } \\
\hline 1 & 169 & 61,0 \\
\hline 2 & 103 & 37,2 \\
\hline 3 & 5 & 1,8 \\
\hline \multicolumn{3}{|l|}{ Afiliación } \\
\hline Vinculado & 10 & 3,6 \\
\hline Subsidiado & 186 & 67,2 \\
\hline Contributivo & 75 & 27,1 \\
\hline No asegurado & 6 & 2,2 \\
\hline
\end{tabular}

Se halló que $225(81,2 \%)$ personas presentaron desconocimiento sobre el chikungunya y 178 (64,3\%) presentaron malas prácticas, posterior a la agrupación y dicotomización de las variables.

No se hallaron diferencias significativas entre el sexo, la edad y la escolaridad y haber presentado la enfermedad. $(\mathrm{p}>0,05)$.

Se hizo un análisis bivariado entre los aspectos sociodemográficos y el conocimiento, se observó que las personas menores de 43 años de edad tienen mayor conocimiento acerca de la enfermedad (72,2\%), aunque no se hallaron diferencias significativas entre el conocimiento de la enfermedad y las variables sexo, edad y escolaridad ( $\mathrm{p}>0,05)$.

Respecto a la escolaridad, se halló que el 30,4\% de los participantes con educación incompleta no conoce en qué se convierte la larva, en comparación con el $17,8 \%$ de los participantes con educación completa; se hallaron diferencias significativas entre el nivel de escolaridad y el ciclo de vida del mosquito (OR 2,01 IC 1,14 a 3,56; $\mathrm{p}=0,015)$. La posibilidad de desconocimiento del ciclo del mosquito en los individuos con escolaridad incompleta es 2 veces en relación con la posibilidad de desconocimiento de los individuos con escolaridad completa.

Según el sexo, el 71,4\% de las mujeres y el 59,7\% de los hombres no conocían las acciones preventivas frente a la enfermedad aunque no se encontró asociación estadísticamente significativa $(\mathrm{p}=0,08)$, de igual forma para la edad y la escolaridad.

El 92,9\% de los participantes que no conocen los síntomas de la enfermedad del chikungunya almacenan agua, en comparación con el 62,0\% de los participantes que sí conocen los síntomas de la enfermedad y almacenaban agua (OR 7,95 IC 1,02-61,9; p=0,001). La posibilidad de desconocimiento de los síntomas de la enfermedad en los individuos que almacenaban agua es 7,95 veces en relación con la posibilidad de los individuos que conocían los síntomas de la enfermedad.

De la misma forma, se encontró asociación estadísticamente significativa entre el desconocimiento de qué es una larva, el ciclo de vida del mosquito y el lugar de reproducción del mosquito según 
el almacenamiento de agua ( $\mathrm{p}=0,001 ; 0,023$ y 0,00 , respectivamente).

Por lo anterior, las personas con desconocimiento de qué es la larva y desconocimiento del ciclo de vida del mosquito, tienen 2 veces la posibilidad de almacenar agua frente a los que conocían qué es la larva y conocen el ciclo de vida del mosquito, así como las personas con desconocimiento del lugar de reproducción del mosquito tienen 3,91 veces riesgo de almacenar agua (Tabla 2).

Los individuos que desconocían el lugar de reproducción del mosquito tienen una asociación estadísticamente significativa respecto al lavado inadecuado de los recipientes $(\mathrm{p}=0,012)$. Presentando una disminución del riesgo de $64 \%$ de lavado inadecuado de recipientes.

Así mismo, el desconocimiento de qué es la larva tiene una asociación estadísticamente significativa con el lavado inadecuado de recipientes $(\mathrm{p}=0,002)$. Presentando dos veces la posibilidad de realizar un lavado inadecuado de los recipientes.

Las personas que sabían a qué hora picaba el mosquito tienen $61 \%$ menos riesgo de no lavar los recipientes en comparación con las personas que no sabían a qué hora picaba el mosquito $(\mathrm{p}=0,01)$ (Tabla 3 ).

Tabla 2. Distribución de conocimientos frente al chikungunya según almacenamiento de agua en el corregimiento de Barcelona, departamento de Quindío, 2016

\begin{tabular}{|c|c|c|c|c|c|c|}
\hline & & \multicolumn{2}{|c|}{ Almacenamiento de agua } & \multirow[b]{2}{*}{$\mathbf{p}$} & \multirow[b]{2}{*}{ OR } & \multirow[b]{2}{*}{ I.C. $95 \%$} \\
\hline & & $\begin{array}{c}\text { Sí } \\
\text { n (\%) }\end{array}$ & $\begin{array}{c}\text { No } \\
\text { n (\%) }\end{array}$ & & & \\
\hline Ha tenido chikungunya & $\begin{array}{l}\text { Sí } \\
\text { No }\end{array}$ & $\begin{array}{l}85(61,1) \\
91(66,4)\end{array}$ & $\begin{array}{l}43(38,9) \\
58(33,6)\end{array}$ & 0,35 & 1,25 & $0,76-2,0$ \\
\hline Qué es chikungunya & $\begin{array}{l}\text { No } \\
\text { Sí }\end{array}$ & $\begin{array}{c}47(55,3) \\
129(67,2)\end{array}$ & $\begin{array}{l}38(44,7) \\
63(32,8)\end{array}$ & 0,05 & 0,6 & $0,35-1,01$ \\
\hline Síntomas de la enfermedad & $\begin{array}{l}\text { No } \\
\text { Sí }\end{array}$ & $\begin{array}{c}13(92,9) \\
163(62,0)\end{array}$ & $\begin{array}{c}1(7,1) \\
100(38,0)\end{array}$ & 0,001 & 7,95 & $1,02-61,9$ \\
\hline Transmisión de la enfermedad & $\begin{array}{l}\text { No } \\
\text { Sí }\end{array}$ & $\begin{array}{c}22(12,5) \\
154(87,5)\end{array}$ & $\begin{array}{l}17(16,8) \\
84(83,2)\end{array}$ & 0,99 & 0,7 & $0,35-1,4$ \\
\hline Lugar reproducción del mosquito & $\begin{array}{l}\text { No } \\
\text { Sí }\end{array}$ & $\begin{array}{c}119(56,9) \\
57(83,8)\end{array}$ & $\begin{array}{l}90(43,1) \\
11(16,2)\end{array}$ & 0,00 & 3,91 & $1,93-7,9$ \\
\hline Conocimiento de la larva & $\begin{array}{l}\text { No } \\
\text { Sí }\end{array}$ & $\begin{array}{c}102(72,3) \\
74(54,4)\end{array}$ & $\begin{array}{l}39(27,7) \\
62(45,6)\end{array}$ & 0,001 & 2,19 & $1,32-3,61$ \\
\hline Conoce metamorfosis de la larva & $\begin{array}{l}\text { No } \\
\text { Sí }\end{array}$ & $\begin{array}{c}51(75) \\
125(59,8)\end{array}$ & $\begin{array}{c}17(25) \\
84(40,2)\end{array}$ & 0,023 & 2,01 & $1,09-3,72$ \\
\hline Hora en que pica el mosquito & $\begin{array}{l}\text { No } \\
\text { Sí }\end{array}$ & $\begin{array}{c}148(61,2) \\
28(80)\end{array}$ & $\begin{array}{c}94(38,8) \\
7(20)\end{array}$ & 0,003 & 0,39 & $0,16-0,93$ \\
\hline Conocimiento de las acciones preventivas & $\begin{array}{l}\text { No } \\
\text { Sí }\end{array}$ & $\begin{array}{c}116(60,7) \\
60(69,8)\end{array}$ & $\begin{array}{l}75(39,3) \\
26(30,2)\end{array}$ & 0,14 & 0,67 & $0,38-1,15$ \\
\hline Conocimiento de la frecuencia de fumigación & $\begin{array}{l}\text { No } \\
\text { Sí }\end{array}$ & $\begin{array}{c}163(62,9) \\
13(72,2)\end{array}$ & $\begin{array}{c}96(37,1) \\
5(27,8)\end{array}$ & 0,42 & 1,53 & $0,52-4,42$ \\
\hline
\end{tabular}


Tabla 3. Distribución de conocimientos frente al chikungunya según lavado de recipientes en el corregimiento de Barcelona, departamento de Quindío, 2016

\begin{tabular}{|c|c|c|c|c|c|c|}
\hline & & \multicolumn{2}{|c|}{ Lavado de recipientes } & \multirow[b]{2}{*}{$\mathbf{p}$} & \multirow[b]{2}{*}{ OR } & \multirow[b]{2}{*}{ I.C. $95 \%$} \\
\hline & & $\begin{array}{c}\text { Sí } \\
\text { n (\%) }\end{array}$ & $\begin{array}{c}\text { No } \\
\text { n (\%) }\end{array}$ & & & \\
\hline Ha tenido chikungunya & $\begin{array}{l}\text { Sí } \\
\text { No }\end{array}$ & $\begin{array}{l}64(43,0) \\
47(36,7)\end{array}$ & $\begin{array}{l}85(57,1) \\
81(63,3)\end{array}$ & 0,35 & 1,25 & $0,47-1,2$ \\
\hline Qué es chikungunya & $\begin{array}{l}\text { No } \\
\text { Sí }\end{array}$ & $\begin{array}{l}32(37,7) \\
79(41,2)\end{array}$ & $\begin{array}{c}53(62,4) \\
113(58,9)\end{array}$ & 0,58 & 1,15 & $0,68-1,95$ \\
\hline Síntomas de la enfermedad & $\begin{array}{l}\text { No } \\
\text { Sí }\end{array}$ & $\begin{array}{c}8(57,1) \\
103(39,2)\end{array}$ & $\begin{array}{c}6(42,9) \\
160(60,8)\end{array}$ & 0,18 & 0,48 & $0,16-1,43$ \\
\hline Transmisión de la enfermedad & $\begin{array}{l}\text { No } \\
\text { Sí }\end{array}$ & $\begin{array}{c}22(12,5) \\
154(87,5)\end{array}$ & $\begin{array}{c}142(85,5) \\
24(14,5)\end{array}$ & 0,82 & 1,08 & $0,53-2,16$ \\
\hline Lugar reproducción del mosquito & $\begin{array}{l}\text { No } \\
\text { Sí }\end{array}$ & $\begin{array}{l}75(35,9) \\
36(52,9)\end{array}$ & $\begin{array}{c}134(64,1) \\
32(47,1)\end{array}$ & 0,012 & 2,01 & $1,15-3,49$ \\
\hline Conocimiento de la larva & $\begin{array}{l}\text { No } \\
\text { Sí }\end{array}$ & $\begin{array}{l}69(48,9) \\
42(30,9)\end{array}$ & $\begin{array}{l}72(51,1) \\
94(69,1)\end{array}$ & 0,002 & 2,14 & $1,31-3,5$ \\
\hline Conoce metamorfosis de la larva & $\begin{array}{l}\text { No } \\
\text { Sí }\end{array}$ & $\begin{array}{l}33(45,8) \\
78(37,3)\end{array}$ & $\begin{array}{c}35(51,5) \\
131(62,7)\end{array}$ & 0,1 & 1,58 & $0,91-2,75$ \\
\hline Hora en que pica el mosquito & $\begin{array}{l}\text { No } \\
\text { Sí }\end{array}$ & $\begin{array}{c}90(37,2) \\
21(60)\end{array}$ & $\begin{array}{c}152(62,8) \\
14(40)\end{array}$ & 0,01 & 0,39 & $0,19-0,81$ \\
\hline Conocimiento de las acciones preventivas & $\begin{array}{l}\text { No } \\
\text { Sí }\end{array}$ & $\begin{array}{l}70(36,7) \\
41(47,7)\end{array}$ & $\begin{array}{c}121(63,4) \\
45(52,3)\end{array}$ & 0,08 & 0,63 & $0,37-1,06$ \\
\hline Conocimiento de la frecuencia de fumigación & $\begin{array}{l}\text { No } \\
\text { Sí }\end{array}$ & $\begin{array}{c}103(39,8) \\
8(44,4)\end{array}$ & $\begin{array}{c}156(60,2) \\
10(55,6)\end{array}$ & 0,69 & 0,82 & $0,31-2,16$ \\
\hline
\end{tabular}

\section{DISCUSIÓN}

Varios países de América y del mundo utilizan las encuestas como una forma de evaluar la participación comunitaria en la prevención de enfermedades. Es importante tener en cuenta en las investigaciones la diversidad geográfica, el nivel educativo y socioeconómico de la población encuestada, además de la presencia de servicios básicos de salud, entre otros.

Los resultados sociodemográficos de esta investigación están en concordancia con estudios anteriores publicados por Nunes et al. ${ }^{13}$, Montero et al. ${ }^{16}$ a diferencia del estudio realizado por Moro et al. ${ }^{17}$ en Villa Romagna (Italia) donde la población correspondió en su mayoría a hombres mayores de 60 años.
Los tres barrios encuestados cuentan con suministro de agua tratada por la empresa de acueducto del municipio de Calarcá, además de recolección de basuras, alcantarillado y el $97,1 \%$ de las calles son pavimentadas. En algunos hogares almacenan agua para el uso en actividades domésticas, esto se considera como un riesgo, ya que no se tienen los cuidados necesarios para evitar formación de criaderos de mosquitos. La empresa de recolección de residuos se encarga además de la limpieza de calles, pero no se realiza mantenimiento en zonas comunes (zonas verdes, quebradas) (observación del autor).

Los conocimientos encontrados muestran que la mayoría de las personas conocen qué es el chikungunya, los síntomas, las formas de prevenirla y cómo se 
transmite la enfermedad, lo que está relacionado con la investigación realizada por Montero et $\mathrm{a}^{16}$ en la ciudad de Bucaramanga, Colombia.

Sin embargo, hay un desconocimiento acerca del ciclo de vida del mosquito, dónde crece, la hora en que pica, la transformación de la larva, las acciones de prevención y la frecuencia de fumigación, algunos de estos resultados son similares a los encontrados en el estudio de Montero et al. ${ }^{16}$

En cuanto al lugar donde obtiene la información acerca de la enfermedad se encontró similitud con el estudio referenciado anteriormente y con el estudio de Moro et al. ${ }^{17}$ en el que la mayoría de las personas obtienen la información a través de medios de comunicación masivos, como radio y $\mathrm{TV}$.

Respecto a las prácticas, la mayoría de personas almacenan agua y los recipientes utilizados con mayor frecuencia son los baldes, esto puede mostrar dificultades con la continuidad de los servicios públicos, lo cual lleva a un aumento en el número de criaderos para el mosquito; además, los participantes dicen que toman precauciones en un $70 \%$, en contraste con la delegación de actividades de prevención de la empresa pública como respuesta a la pregunta sobre la importancia de las acciones en el barrio.

En caso de tener fiebre la mayoría de las personas refirió auto medicarse, y consultar al centro de salud de la localidad en un segundo lugar. Esta práctica es bastante preocupante, ya que existe la posibilidad de que el manejo de la patología sea el inadecuado, aumentando el riesgo de complicaciones e incapacidades prolongadas, además de ello, anula la posibilidad de notificación de hacer seguimiento y control para evitar la transmisión (observación del autor).

Las prácticas anteriormente relacionadas favorecen la continuidad de la cadena de transmisión de las arbovirosis y las presentaciones endémicas de la enfermedad ${ }^{14,18}$, esto de igual forma nos indica que el tener conocimientos adecuados no necesariamente se traduce en buenas prácticas preventivas como lo señala Benítez et al. ${ }^{19}$.

La percepción de los participantes frente a la falta de acciones preventivas por la comunidad a la que pertenecen corresponde en $52 \%$ por pereza y en el $41 \%$ por desinterés en el tema. Lo cual es acorde con los hallazgos de Montero et al. ${ }^{16}$ Con esto se muestra una falta de empoderamiento de la población frente a las actividades preventivas para el control de las enfermedades vectoriales esto en concordancia con el estudio realizado por Vilcarromero et al. ${ }^{20}$, ya que no hay una motivación clara para realizarlas, lo que a su vez muestra la falta de acciones de intervención de los entes administrativos sobre estas comunidades ${ }^{18,19}$.

Los hallazgos encontrados en esta investigación muestran que a pesar de la presencia de chikungunya en los barrios encuestados y de la ocurrencia de brotes epidémicos frecuentes, los conocimientos son deficientes. Estos hallazgos difieren de los divulgados por Montero et al. ${ }^{16}$, donde encontraron conocimientos adecuados sobre el chikungunya.

Esta diferencia puede deberse a que la encuesta del estudio de Bucaramanga se realizó tras haber establecido planes de control a la comunidad, debido a la epidemia de dengue clásico reportada en el departamento para el 2013 y 2014. Esta explicación es consistente con los hallazgos de Dowling et al. ${ }^{23}$ en los que reportan que personas procedentes de zonas epidémicas o donde hay mayor riesgo de sufrir la enfermedad, tienen mejor nivel de conocimiento sobre esta.

Al comparar las características sociodemográficas con los conocimientos, no encontramos diferencias estadísticamente significativas entre estas variables. Contrario con lo hallado en Washington en el estudio de Dowling et al. ${ }^{23}$, en las que las personas de mayores ingresos y en edad joven presentaron mejor conocimiento y las personas mayores y menos educadas perciben en mayor cantidad la gravedad de la enfermedad. Las personas mayores de 46 años con educación avanzada tienen mayor probabilidad de conocer el desarrollo del mosquito y la reducción de los criaderos. Mientras que lo hallado en el estudio de Moro et al. ${ }^{17}$ suguiere una asociación entre las personas jóvenes y el menor conocimiento.

De igual manera, en el análisis estadístico no se encontró asociación entre los aspectos sociodemográficos frente las prácticas. Por el contrario, en el análisis de los conocimientos frente a las prácticas se relacionaron las variables conocimiento de los síntomas de 
la enfermedad, lugar de reproducción del mosquito, conocimiento de la larva, conocimiento de la metamorfosis de la larva y hora en que pica el mosquito con el almacenamiento de agua con una asociación estadísticamente significativa entre estos resultados.

Esto refleja que los conocimientos de los participantes influencian directamente las prácticas que ponen en riesgo su salud, mostrándonos la importancia de estos hallazgos para así encaminar las acciones de intervención en la comunidad. Lo anterior en concordancia con el estudio de Dowling et al. ${ }^{23}$ en el cual la práctica de eliminación de los criaderos tiene mayor probabilidad en individuos con mayor conocimiento del ciclo de vida del mosquito.

Se identificó como asociado a un menor riesgo el conocimiento de la hora en que pica el mosquito, con una reducción de $61 \%$ y como asociados a un mayor riesgo el lugar de reproducción del mosquito y el conocimiento de la larva. No se encontró literatura relacionada con los anteriores hallazgos, como también, la limitación de desarrollarse con una muestra por conveniencia, por lo que los resultados aquí planteados $y$ las inferencias deben ser analizados con prudencia.

\section{CONCLUSIÓN}

Los conocimientos y las prácticas en el corregimiento de Barcelona, departamento del Quindío frente al chikungunya son deficientes, esto relacionado con la carencia de canales de información locales adecuados y la falta de programas de control y eliminación de vectores.

Los medios de comunicación se han hecho presentes en brotes o alarmas, pero se encuentra casi ausente en los periodos de silencio epidemiológico, etapas en las que se debería reforzar la información brindada en momentos de alarma.

Se evidencia una falta de empoderamiento de la comunidad en temas como el control vectorial, puesto que se observan criaderos en sus domicilios, no están atentos a planes de erradicación, además de no prestar la suficiente atención a la poca información que circula.
Es de vital importancia que al ser Colombia un país tropical, la educación sobre enfermedades producidas por vectores se haga a través de todos los medios disponibles con lenguaje adecuado por regiones, con un apoyo financiero sostenible, de igual manera, enfatizar la educación en los niños, estableciendo logros a largo plazo, la organización de foros comunitarios en los que puedan participar líderes, representantes de iglesias, colegios y gobiernos locales, para difundir información sobre la epidemia y las acciones de prevención, además de realizar sondeos y retroalimentaciones constantes.

\section{Agradecimientos}

A las personas que participaron y a las encuestadoras: Mónica y Johana que facilitaron la labor.

\section{REFERENCIAS}

1. Ministerio de Salud y Protección Social. Plan de Salud de respuesta frente a la fiebre por el virus chikungunya. 2014, agosto. [Internet] Recuperado a partir de: https:// www.minsalud.gov.co/sites/rid/Lists/BibliotecaDigital/ RIDE/VS/PP/ET/PLAN\%20NACIONAL\%20DE\%20 RESPUESTA\%20CHIKUNGUNYA\%20COLOMBIA

2. Organización Mundial de la Salud. Chikungunya. Nota descriptiva $\mathrm{N}^{\circ} 327$. [Internet]; mayo 2015 [citado 2015 Junio 11]. Recuperado a partir de: OMS.URL disponible en: http://www.who.int/mediacentre/factsheets/fs327/es.

3. Clouet-Huerta D, Alfaro-Toloza P, Rodríguez-Morales AJ. Chikungunya en las Américas: Preparación, vigilancia y alerta en Chile. Rev Chilena Infectol. 2014;31(6):761762. doi: 10.4067/S0716-10182014000600022.

4. Weaver SC. Arrival of chikungunya Virus in the new World: prospects for spread and impact on Public Health. PLoS Negl Trop Dis. 2015;8(6):e2921. doi: 10.1371/journal.pntd.0002921.

5. Rey JR, Lounibos P. Ecología de Aedes aegypti y Aedes albopictus en América y trasmisión enfermedades. Biomédica. 2015;35:177-85. doi: 10.7705/biomedica.v35i2.2514.

6. Alcalá L, Quintero J, González-Uribe C, Brochero H. Productividad de Aedes aegypti (L.) (Diptera: Culicidae) en viviendas y espacios públicos en una ciudad endémica para dengue en Colombia. Biomédica. 2015;35(2):258-68. doi: https://doi.org/10.7705/biomedica.v35i2.2567. 
7. Figueiredo MLG, Figueiredo LTM. Emerging alphaviruses in the Americas: Chikungunya and Mayaro. Rev Soc Bras Med Trop. 2014; 47(6): 677-683. doi: 10.1590/0037-8682-0246-2014.

8. Organización Panamericana de la Salud. Preparación y respuesta ante la eventual introducción del virus del ckikungunya en las Américas [Internet]. Octubre 2011; Recuperado a partir de: http//:http://wwwl.paho.org/hq/ dmdocuments/CHIKV_Spanish.pdf

9. Instituto Nacional de Salud. Boletín epidemiológico semanal. Semana epidemiológica 52 de 2015. [Internet]. 2015, noviembre; Recuperado a partir de: http://www.ins. gov.co/boletin-epidemiologico/Paginas/default.aspx

10. Instituto Nacional de Salud. Boletín epidemiológico semanal. Semana epidemiológica 15. 2016. [Internet]. 2016, abril. Recuperado a partir de: http: // www.ins.gov.co/boletin-epidemiologico/Paginas/default.aspx

11. Placeres Hernández JF, Martínez Abreu J, Chávez González L, Rodríguez Rodríguez E, De León Rosales L. Fiebre causada por el virus chikungunya, enfermedad emergente que demanda prevención y control. Rev. Med. Electrón. [Internet]. 2014;36(5):596-609. Recuperado a partir de: http://scielo.sld.cu/scielo.php?script=sci_arttext\&pi$\mathrm{d}=$ S168418242014000500007\&lng=es.

12. Cáceres-Manrique FM, Vesga-Gómez C, Perea-Florez X. Conocimientos, actitudes y prácticas sobre dengue en dos barrios de Bucaramanga, Colombia. Rev. Salud Pública Bogotá. 2009;11(1):27-38. doi: 10.1590/ S0124-00642009000100004.

13. Nunes M, Rodríguez N, Mota J, Golding N, Kramer M, Freitas L et al. Emergence and potential for spread of chikungunya virus in Brazil. BMC Med. 2015;13:102. doi: 10.1186/s12916-015-0348-x.

14. Espinoza-Gómez F, Hernández-Suárez CM, Coll-Cárdenas R. Educational campaign versus malathion spraying for the control of Aedes aegypti in Colima, Mexico. J Epidemiol Community Health. 2002;56(2):148-152. doi: 10.1136/jech.56.2.148.
15. Registro Hospital Centro de Salud Barcelona sede de E.S.E. Hospital La Misericordia, Barcelona, Calarcá, Quindío.

16. 16. Montero Y, Zapata G, Moreno J. Conocimientos, actitudes y prácticas sobre el chikungunya en los habitantes del barrio San Martin Alto, de la comuna Nueve de la ciudad de Bucaramanga. Colombia. Aplicatec Electrón. [Internet]. 2015;5(1). Recuperado a partir de: http:// www.fitecvirtual.org/ojs-3.0.1/index.php/FITEC/article/ view/156.

17. 17. Moro ML, Gagliotti C, Silvi G, Angelini R, Sambri V, Rezza G, et al. Knowledge, attitudes and practices survey after outbreak of chikungunya infection. Int Health. 2010;2(3):223-227. doi: 10.1016/j.inhe.2010.07.003.

18. Pineda F, Agudelo CA. Percepciones, actitudes y prácticas en malaria en el Amazonas colombiano. Rev Salud Pública. Bogotá. 2005;7(3):339-48.

19. Benítez-Leite S, Machi ML, Gibert E, Rivarola K. Conocimientos, actitudes y prácticas acerca del dengue en un barrio de Asunción. Rev. chil. pediatr. 2002;73(1):64-72.

20. Vilcarromero S, Casanova W, Ampuero JS, Asayag C, Siles C, Díaz G, et al. Lecciones aprendidas en el control de Aedes aegypti para afrontar el dengue y la emergencia de chikungunya en Iquitos, Perú. Rev Perú Med Exp Salud Pública. 2015;32(1):172-8.

21. Ministerio de Salud del Perú. Dirección General de Promoción de la Salud. Proyecto Vigía. Conocimientos, actitudes y prácticas de la población frente al dengue: Lima y Macro. Norte. Lima, Perú. 2004;138.

22. Rodríguez H, De la Hoz FP. Dengue and vector behavior in Cáqueza, Colombia, 2004. Rev. Salud Pública, Bogotá. 2005;7(1):1-15.

23. Dowling Z, Armbruster P, Ladeau SL, Decotiis M, Mottley J, Leisnham PT. Linking Mosquito Infestation to Resident Socioeconomic Status, Knowledge, and Source Reduction Practices in Suburban Washington, D.C. Eco health. 2013;10(1):36-47. doi: 10.1007/s10393-013-0818-6. 